\title{
A 40-yr-old male with cough, haemoptysis and increasing dyspnoea
}

\author{
G.C. Riise*, M. Bugge ${ }^{\#}$, Å.A. Johnsson*, H. Willén ${ }^{+}$
}

\section{Case report}

A 40-yr-old Caucasian male was referred to the Dept of Respiratory Diseases because of cough and increasing dyspnoea. He had been through several courses of antibiotics for these symptoms as well as periods of fever during the last 12 months. In spite of this, his nonproductive cough had increased and finally resulted in a haemoptysis 2 weeks prior to the investigation. Weight loss and lack of physical strength were also present at this time.

He was a lifelong nonsmoker, lived in the countryside and worked as a carpenter. He had previously been diagnosed with pulmonary sarcoidosis in 1992, and had been treated with tapering doses of peroral corticosteroids and controlled without recurrence of the disease. He is now on no regular medication.

Routine physical examination did not show any physical abnormalities. Laboratory investigations revealed blood gas values within the normal range, a slight anaemia (haemoglobin $126 \mathrm{~g} \cdot \mathrm{L}^{-1}$ ) and a blood leukocytosis of $14.3 \times 10^{9} \cdot \mathrm{L}^{-1}$ (mainly neutrophils, $87 \%$ ). Serum level of angiotensin converting enzyme was normal, C-reactive protein was increased to
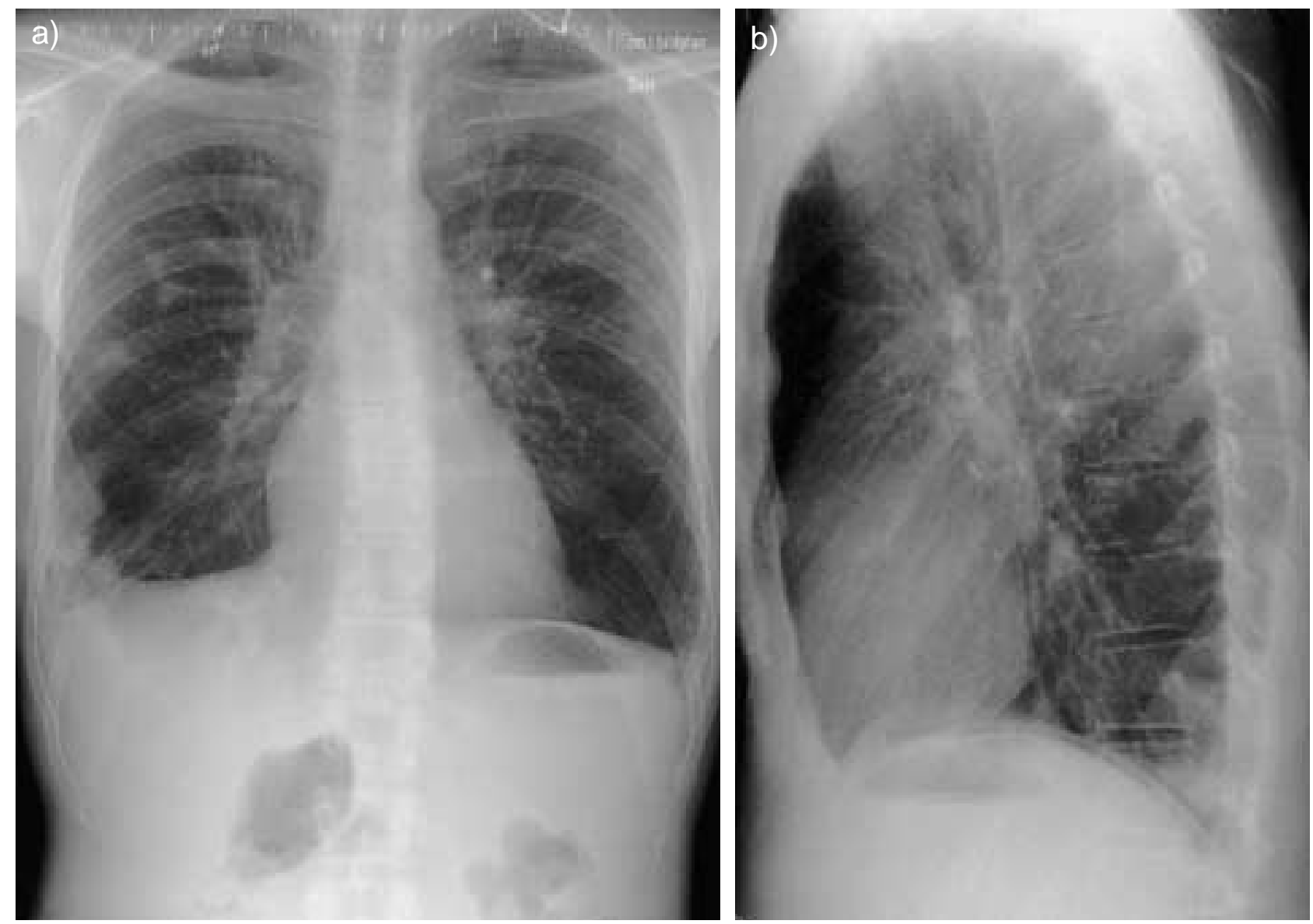

Fig. 1.-Chest X-ray a) frontal and b) side projection.

*Dept of Respiratory Medicine, ${ }^{\#}$ Dept of Cardiothoracic Surgery, ${ }^{\text {DDept }}$ of Thoracic Imaging, ${ }^{+}$Dept of Pathology, Sahlgrenska University Hospital, University of Göteborg, Sweden.

Correspondence: G.C. Riise, Dept of Respiratory Medicine, Sahlgrenska University Hospital, S-413 45 Göteborg, Sweden. Fax: 4631824904. 
$76 \mathrm{mg} \cdot \mathrm{L}^{-1}$, and the blood sedimentation rate slightly increased to $22 \mathrm{~mm} \cdot \mathrm{h}^{-1}$. Blood analyses of rheumatoid factor, antinuclear antibodies, antineutrophil cytoplasmatic antibodies, smooth muscle antibodies and CD4+/CD8+ ratios were normal. Repeated sputum samples for tuberculosis were negative.

Electrocardiogram revealed sinustachycardia with unspecific T-wave changes anteriorly. A chest radiograph (CXR) was taken (fig. 1a, b).

The patient underwent bronchoscopy and bronchoalveolar lavage (BAL), but no transbronchial biopsies of the CXR changes were attempted due to severe cough. Macroscopically, only a slight mucosal oedema was noted in the bronchial tree, and the BAL cell viability and cell differential counts were normal. A significant growth of Streptococcus pneumoniae found in BAL cultures was treated adequately with penicillin.

Computed tomography (CT) as well as magnetic resonance imaging (MRI) were performed (figs. 2 and 3 ), and the findings resulted in the patient undergoing a thoracotomy. Lung biopsy of the intrathoracic densities was performed (fig. 4).

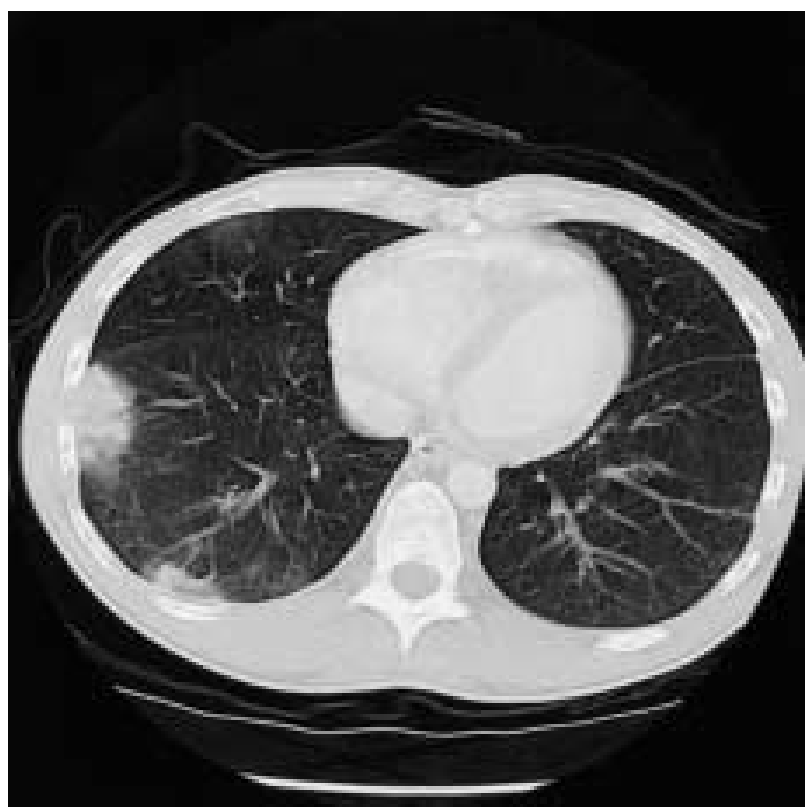

Fig. 2.-Computed tomography of the thorax.

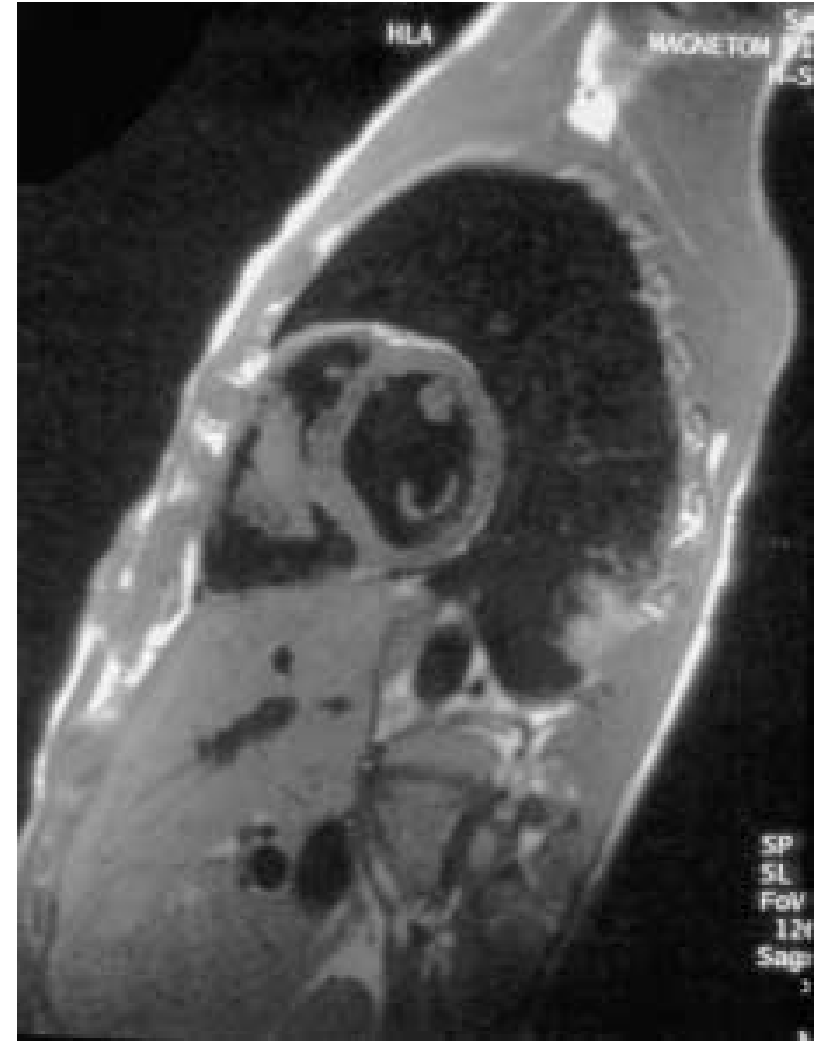

Fig. 3.-Magnetic resonance image of the thorax.

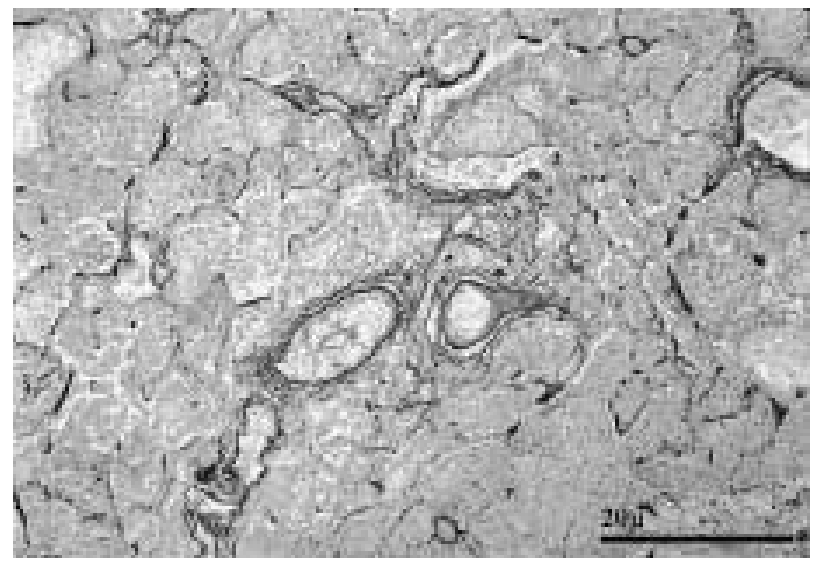

Fig. 4. - Lung biopsy, van Gieson stain, scale bar=20 $\mu \mathrm{m}$.

BEFORE TURNING THE PAGE, INTERPRET THE CXR (FIG. 1), CT (FIG. 2), MRI (FIG. 3) AND THE LUNG BIOPSY PATHOLOGY (FIG. 4) AND SUGGEST A DIAGNOSIS. 


\section{Interpretation}

\section{Chest radiography}

Figure 1a and $\mathrm{b}$ shows normal heart size, several well-defined rounded, densities in the left upper lobe as well as right lower lobe, and a larger inflammatory opacity at the base of the right lung.

\section{Computed tomography}

The helical CT in figure 2 depicts triangular densities with broad base towards the pleura in the lower lobes indicating possible pulmonary infarctions. The major arteries were free of thrombi. On other sections, rounded densities $(1-1.5 \mathrm{~cm})$ were found in the upper lobes, and small lymph nodes were present in the mediastinum. In retrospect, a mass was suspected in the right ventricle.

\section{Magnetic resonance imaging}

The cardiac MRI examination (fig. 3) clearly demonstrates a lobular mass arising on a stem from the interventricular septum in the right ventricle. The tumour was surrounded by thrombotic material, and parts of the papillary muscles were embedded in the mass. The tricuspid valve was intact.

\section{Pathology}

The lung biopsy was $5 \times 3 \times 2 \mathrm{~cm}$ large, with a wedgeshaped, dark haemorrhagic zone of a pulmonary infarction (fig. 4). Microscopically, there was a sharp border between the infarction and the normal tissue. The alveolar spaces were filled with haemorrhage, oedema fluid and degenerated cells. The pulmonary vessels supplying the area of infarction were obliterated by emboli composed of myxoid matrix with few or no cells. In some vessels, there were signs of recanalization (fig. 4).

\section{Diagnosis: "Right ventricle myxoma causing multiple pulmonary tumour emboli"}

\section{Clinical course}

The patient underwent both open heart surgery and open lung biopsy of one of the palpable intrapulmonary masses. The lobulated myxoma in the right ventricle originated from the interventricular septum, and was surrounded by thrombotic material. A biological tricuspid valve prosthesis had to be inserted when radically excising the myxoma, since resectioning of the papillary muscles was necessary.

At follow-up, 4 months after the operation, the patient was clinically well. CT scan demonstrated that some of the pulmonary infarctions had resolved, but several rounded densities, which were interpreted as possible metastases, persisted. MRI detected no recurrence of the intracardiac tumour.

\section{Discussion}

Cardiac tumours are rare, with an incidence of $<0.1 \%$ in autopsy materials. Benign myxomas account for about two-thirds of these, and they are found in the left atrium in $75 \%$ of the patients, in the right atrium in $20 \%$, and rarely in the right ventricle [1]. Altogether, there have been 10 case reports of right atrial myxomal tumour as a cause for embolism to the lung [2]. These patients had a wide range in age (18-61 yrs), and the diagnosis was mostly made with angiogram or echocardiogram.

Unfortunately, delay in diagnosing this disease is common. In one series, the mean duration from onset of symptoms to diagnosis was well over 10 months [3]. This was also true for the patient in the present study who had complained of several symptoms, albeit nonspecific, for over a year. Extracardiac symptoms commonly associated with myxoma are dyspnoea, fatigue, and low grade fever. Laboratory findings include anaemia, leukocytosis and an elevated sedimentation rate, all of which were present in this patient. In addition, increased serum levels of gamma globulin and a risk for development of disseminated intravascular coagulation can be seen [4]. Recently, interleukin-6 has been associated with myxomas, and production of this cytokine could cause many of the accompanying inflammatory or autoimmune symptoms [5].

Since myxomas are fragile and gelatinous in their structure, embolization can occur quite easily and can give rise to additional symptoms. These are mostly systemic since the majority of myxomas are found in the left atrium. However, recurrent pulmonary embolism without any detectable source of thrombosis is a clinical situation where cardiac myxoma on the right side should be considered. Fortunately for the patient, the progress of his tumour growth and pulmonary embolization had not been fatal considering the long period that preceded the final diagnosis. The authors initially suspected that the patient's complaints were due to atypical lung infections based on possible underlying immunodeficiency, or Wegener's granulomatosis, hence the BAL investigation. It was not until both CT scan and MRI were performed that the final diagnosis of cardiac myxoma with possible pulmonary embolism emerged.

The treatment is always surgical and urgent once the diagnosis has been established. Radical excision is often curative in nonfamilial myxomas, although long-term clinical follow-up should always be aimed for to rule out recurrence [4]. After successful surgery, magnetic resonance imaging should be used for serial follow-up, since it can detect early recurrence of tumours [1]. The patient still had several rounded densities on computed tomography at follow-up, which were interpreted as remaining metastases. These findings, of course, have to be monitored in the future. Remote growth of myxomatous material 
had been observed, indicating a semimalignant course in an otherwise benign disease [6].

\section{References}

1. Reynen K. Cardiac myxomas. N Engl J Med 1995; 333: $1610-1617$.

2. Idir M, Oysel N, Guibaud JP, Labouyrie E, Roudaut R. Fragmentation of a right atrial myxoma presenting as a pulmonary embolism. $J$ Am Soc Echocardiogr 2000; 13: 61-63.

3. Goswami KC, Shrivastava S, Bahl VK, Saxema A,
Manchanda SC, Wasir HS. Cardiac myxomas: clinical and echocardiographic profile. Int J Cardiol 1998; 63: 251-259.

4. McCoskey EH, Mehta JB, Krishnan K, Roy TM. Right atrial myxoma with extracardiac manifestations. Chest 2000; 118: 547-549.

5. Seino Y, Ikeda U, Shimada K. Increased expression of interleukin-6 mRNA in cardiac myxomas. Br Heart $J$ 1993; 69: 565-567.

6. Diflo T, Cantelmo NL, Haudenschild CC, Watkins MT. Atrial myxoma with remote metastasis: case report and review of the literature. Surgery 1992; 111: 352-356. 